\title{
Die Trommel ruft - die Banner wehn
}

oder: wie die KPD bei Osram streikte

Klassenkampf, Betriebsgruppe bei Osram Westberin

Dies soll kein Bericht sein über die Lage in einem bestimmten Betrieb oder über die Arbeit unserer Betriebsgruppe, sondern wir wollen an einem konkreten Beispiel, das sich bei Osram/Westberlin Mitte November ereignet hat, die verantwortungslose und falsche Politik der sogenannten „KPD“ darstellen. Das scheint uns umso wichtiger, als die KPD-AO und ihre ,Rote Hilfe e. $\mathrm{V} .{ }^{6}$ in ihren Zeitungen und Flugblättern mit ihren Osram-Erfolgen hausieren gehen und so ahnungslosen Kollegen und ,Menschen' (die AO trennt in ihrer Propaganda sorgfältig zwischen Arbeitern und Menschen $=$ Studenten!) vorgaukeln, welch aktive und schlagkräftige Organisationen sie seien. Wir halten es auch für lehrreich, die Auseinandersetzung mit der AO nicht nur in der Theorie, sondern auch mal anhand eines konkreten Vorfalls zu führen.

\section{Die Lage im Westberliner Osram-Werk}

Wir arbeiten in dem neuen Entladungslampenwerk, das Osram mit finanzieller Un. terstützung des Berliner Senats vor rund einem Jahr in Spandau fertigstellte. Die Situation ist für die Kollegen seit dem Umzug in dieses neue Werk noch viel schlechter als im alten Werk: Die gesamte Produktion vollzieht sich in einer riesigen Halle, die durch keine Zwischenwände unterteilt ist. Lärm, schlechte Klimaanlagen machen das Arbeiten unerträglich. Die sozialen Einrichtungen sind mehr als dürftig, der Werksgesundheitsdienst ist räumlich und personell völlig unzureichend.

Dazu kommt, daß das Arbeitstempo ständig gesteigert wird. Teils laufen die Maschinen schneller, teils werden Kollegen eingespart (man stellt seit längerer Zeit fast nur noch Facharbeiter ein, obwohl viele Kollegen von diesem ,modernsten Lampenwerk Europas" die Schnauze voll haben und abhauen), oder auch beides. Kurz, das Arbeitsklima ist so beschissen, wie es nicht mehr schlimmer vorzustellen ist.

Wir arbeiten im 2 Schicht-Betrieb. Zur Zeit sind wir ca. 1000 Produktionsarbeiter, darunter weit über die Hälfte Frauen. Der Anteil der Ausländer beträgt über $50 \%$. Vor allem arbeiten bei uns Türken (ca. 500, also $50 \%$ ), ca. 80 Jugoslawen und eine kleine Anzahl von Griechen, Italienern und Spaniern. Die Struktur ist, wie meistens in der Elektroindustrie, folgende: An den einzelnen Fertigungslinien arbeiten relativ wenige und gutbezahite Einrichter (zwischen 8,50 und 11 DM die Stunde), die zum größten Teil Facharbeiter sind, vor allem Deutsche und Jugoslawen. Dazu kommt dann die große Masse von Bandarbeitern, die im Schnitt um 6 DM verdienen, überwiegend die Türken, bei den Bandarbeiterinnen, die $z$ wischen 4,60 und 6 DM verdienen, überwiegen die Türkinnen und ältere deutsche Frauen. 
Diese Zusammensetzung der Belegschaft machte es im alten Werk, in dem die einzeinen Abteilungen zudem räumlich voneinander getrennt waren, schwierig, zu gemeinsamen Aktionen oder überhaupt zu einem solidarischen Verhalten der Kollegen untereinander zu kommen. Die Spaltung in gut bezahlte Einrichter und schlecht bezahlte Bandarbeiter, zwischen Deutschen und Ausländern, und zwischen verschie. denen ausländischen Nationalitäten verhinderte das weitgehend.

Diese Situation veränderte sich, sobald der Umzug ins neue Werk stattfand und alle Kollegen gemeinsam den neuen menschenfeindlichen Bedingungen in der neuen Fabrik ausgesetzt waren. Außerdem brachte der Umzug ins neue Werk, der sich fließend und möglichst ohne Unterbrechung der Produktion vollziehen sollte, zusätzliche Arbeitsbelastungen und Umstellungen für die Kollegen mit sich.

Von Anfang an zeigte sich dadurch und durch die verbesserten Kommunikationsmöglichkeiten (im neuen Werk arbeiten alle zusammen in einer Halle, im alten waren wir räumlich voneinander isoliert) ein verändertes Verhältnis der Kollegen zueinander, das sich in ersten und bescheidenen Widerstandsformen gegen die schlecht funktionierende Klimaanlage und gegen die Lärmbelästigung ausdrückte. Hinzu kamen die allgemein schlechteren Lebensbedingungen für die Arbeiter durch den viel zu niedrigen Tarifabschluß im Januar 73 und die ansteigenden Lebenshaltungskosten, die in Westberlin noch um rund $1 \%$ höher stiegen als in Westdeutschland. Dieses Zusammentreffen zweier Faktoren - die miesen Arbeitsbedingungen in der neuen Fabrik und der allgemeine Lohnraub - brachte zum ersten Mal seit mehreren Jahren eine Bewegung zwischen den Osram-Kollegen zustande, die die Spaltung der Kollegen wenigstens teilweise überwand und zu der gemeinsamen Forderung nach einer Teuerungszulage führte. Diese Forderung wurde auf einer Betriebsversammlung Ende Juni aufgestellt und von allen Kollegen als Verhandlungsauftrag an den Betriebsrat weitergegeben. Durch die Urlaubszeit war es der Geschäftsleitung und auch dem Betriebsrat jedoch ein leichtes, diese Forderung stillschweigend unter den Tisch fallen zu lassen und sie ohne größeren Protest der Kollegen abzulehnen.

Nach dem Urlaub - in dieser Zeit liefen auch in Westdeutschland die wilden Streiks - wurde das jedoch anders, zumal immer mehr Kollegen ins neue Werk umziehen mußten. Wir wollen jetzt nicht alle Einzelheiten darstellen. Wichtig ist, daß es wegen der geforderten Teuerungszulage zu einem kleinen Warnstreik kam, daß eine Unterschriftensammlung durchgeführt wurde ( 600 Kollegen unterschrieben trotz Behinderungen durch die Meister) und daß für den Fall, daß eine Teuerungszulage von 300 DM) nicht bis Anfang Oktober gezahlt würde, die Kollegen sich auf einen Streik vorbereiteten. In dieser Bewegung waren sowohl türkische wie jugoslawische und deutsche Kollegen aktiv, d. h. die Forderung wurde als gemeinsame aller Kollegen verstanden. In dieser Bewegung zeigte sich aber auch, daß viele der deutschen gutbezahlten $F$ acharbeiter am wenigsten solidarisch waren. Somit ergab sich innerhalb der Belegschaft auch eine Polarisierung, die unter anderem dazu führte, daß der Einfluß dieser Facharbeiter, die in vielen $F$ ällen als Obereinrichter an den Fertigungslinien faktisch Vorarbeiterfunktionen innehatten, auf ihre Kollegen, auch auf schlechter bezahlte deutsche Einrichter, rapide abnahm. Sie hatten sich bei der gemeinsamen Forderung nicht auf die Seite der Kollegen gestellt, und waren damit bei den Kollegen ,unten durch". 
Somit war die Spaltung der Kollegen untereinander zwar nicht völlig abgebaut, aber der unsolidarische Teil der Kollegen war relativ klein und bekannt. Die Mehrzahl der Kollegen, auch der deutschen, war sich einig, diese Forderung nach einer Teuerungszulage durchzusetzen.

Dieser gemeinsamen Bewegung versuchte der Betriebsratsvorsitzende, sicherlich in Einvernehmen mit der Geschäftsleitung, dadurch entgegenzuwirken, daß er die entscheidende Betriebsversammlung Anfang Oktober, auf der die Geschäftsleitung ja oder nein zu unserer Forderung sagen mußte, aufspalten wollte. Er setzte eine gatrennte Betriebsversammlung zur selben Zeit für die Türken an, wohl in der Annnahme, die Türken würden sich an einem nach A blehnung einer Teuerungszulage eventuell ausbrechenden Streik als geschlossenste und kämpferischste Gruppe beteiligen. Daß die Türken nicht allein losschlagen würden, war klar. Sie haben immer wieder betont: ,Nur zusammen mit den deutschen Kollegen; Nicht wie bei FORD! “.

Sofort nach Bekanntwerden dieser Spaltungsabsichten ging im Betrieb unter

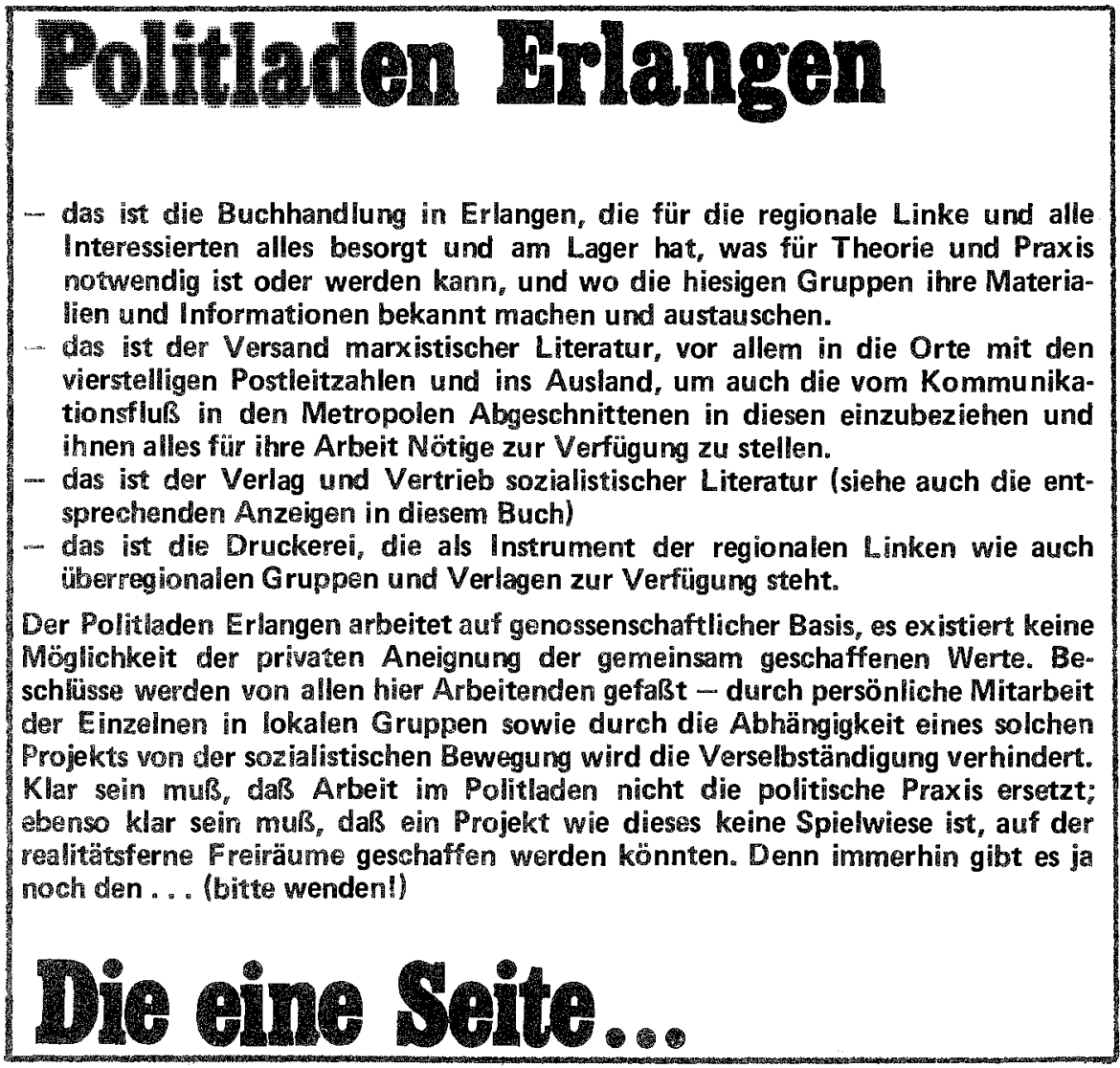


allen Kollegen, nicht nur unter den türkischen, die Parole um:, Wir haben gemeinsame Probleme und gemeinsame Forderungen, also brauchen wir auch eine gemeinsame Versammlung. " Deutsche, tiirkische, jugoslawische, griechische Kollegen waren sich so einig wie nie zuvor. Obwohl weiterhin eine kleine Gruppe von älteren deutschen Frauen und deutschen Einrichtern nicht mitzog und sogar unwillig wurde, als auf der erzwungenen gemeinsamen Versammlung längere Redebeiträge in türkisch gehalten wurden und zwischendurch übersetzt werden mußten. Diese Spaltung wäre höchstens nach Ausbruch eines erfolgversprechenden Streiks zu überwinden gewesen, vielleicht auch nicht einmal dann. Jedenfalls stand sie einem Streik nicht mehr im Wege, da sie eine Spaltung zwischen einer hartnäckig ausländerfeind. lichen und betriebshörigen Minderheit und der kampfbereiten großen Mehrheit der Belegschaft war.

Außerdem verhielten sich die Türken untereinander zum ersten Mal wie eine solidarische Gruppe. Weil sie den Plan des Betriebsrats durchschauten, fühlten sie sich in ihrem Selbstbewußtsein bestärkt. Sie merkten, daß es bei Osram vor allem

\section{mognslth nobsitiloq}

... Kapitalismus! Das heißt für den Politladen, so zu arbeiten, daß er als Produktions- und Handelsbetrieb existenzfähig ist. Das heißt Mehrwertschaffung, Akkumulation, Kalkulation zu kostendeckenden Preisen, Buchführung, Finanzamt, Versicherung. Werbung, Entfremdung, Lohnlerhöhung, wenn auch für alle gleich und überhaupt recht durchschnittlich). Anders $2 \mathrm{u}$ ",wirtschaften ${ }^{20}$ hieße technische und organisatorische Mittel für die sozialistische Bewegung faktisch aufzugeben, da der Konkurs nur eine Frage der Zeit wäre.

Diese unumgänglich notwendige Art des Arbeitens stößt aber auf ein erhebliches Hindernis: den Kapitalmangel. Wenn man ohne Kapital anfängt und gleichzeitig versucht, den ganzen Laden auszubauen ${ }_{g}$ damit er leistungsfähiger wird und auch Krisen und Repressionen kleineren Umfangs ihn nicht umwerfen, bleibt nichts zum akkumulieren. Hauptschwierigkeit: Alle Kosten müssen bezahlt werden, bevor das Geld aus dem Verkauf an Buchhandlungen, Verlage, Versandkunden usw. eintrifft, so daß ständig ein großes Finanzloch - verursacht durch diese Zeitdifferenz - entsteht. " ${ }_{\nu}$ Normale ${ }^{\prime A}$ Firmen nehmen dann die Kredite der Banken in Anspruch, bei uns klappte das nie. Zum einen haben wir die Grundstücke nicht, die als Sicherheiten gewünscht wären, zum anderen ist die Geldgier der Banken nicht immer so groß, daß sie ihren eigenen Untergang aurch noch finanzieren möchten. Daher die dringende Bitte an alle Genossinnen und Genossen, Freunde und Leser(!): Statt Sparbücher anzulegen uns Darlehen zu gewähren! 100 Linke mit $500 \mathrm{DM}$ oder 50 mit $1.000 \mathrm{DM}$ müßs ten doch schaffen, was die eine Bank mit 50.000 nicht will! Schreibt uns, wenn Ihr solche Kreditmöglichkeiten seht, wir schicken Euch einen Vertrag (Kündigungsfristen, Zinsen usw.) und mehr Informationen. Bitte bald reagieren, bevor es zu spät ist. Schreibt an den Politladen, 8551 Gaiganz.

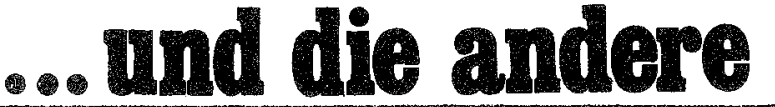


auf ihr Verhalten ankommt. Dadurch wurde zum Beispiel die Trennung zwischen zwischen tïrkischen Männern und Frauen, die vorher ein gemeinsames Vorge. hen und Verständnis der Türken mit verhindert hatte, abgebaut. Der Erfolg war, daß auf der Extra-Türken-Veranstaltung 10 Betriebsräte und Vertreter der Geschäftsleitung und nur 3 türkische Kollegen dawaren. Der Rest war in der gemeinsamen Betriebsversammlung und beteiligte sich lautstark und selbstbewußt an der Diskussion.

Der nach der Betriebsversammlung mögliche und vorher von den Kollegen abgesprochene Streik kam nicht zustande, weil die Geschäftsleitung auf der Versammlung die Zahlung einer einmaligen Zulage von $200 \mathrm{DM}$ bekannt gab, und die Mehrheit der Kollegen nicht bereit war, für die 100 DM Differenz zu streiken, obwohl sie unzufrieden mit der niedrigen Zulage waren.

Die zunehmende Solidarisierung aller Kollegen, die auch eine Politisierung und ein größeres Selbstbewußtsein der Kollegen zur Folge hatte, bewies sich auch bei den Vertrauensleutewahlen, die in den wichtigsten und größten Abteilungen des neuen Werks wegen des stufenweisen Umzugs erst im November abgehalten wurden. Ein großer Teil der Kollegen wählte bewußt solche Vertrauensleute, die sich bei den vorhergehenden Aktionen für eine Teuerungszulage hervorgetan hatten. Das war auch als Antwort an den alten und immer noch amtierenden Betriebsrat zu verstehen, der nur kleinlaute Bittbriefe geschrieben hatte und in dem je ein Alibi-Türke und -Jugoslawe sitzt. Zum ersten Mal wurden ausländische Kollegen zu gewerkschaftlichen Vertrauensleuten gewählt: 7 Türken, 1 Jugoslawe, 1 Grieche.

In dieser Bewegung unter den türkischen Kollegen spielte auch der neue Dolmetscher, Mukadder C., eine vorantreibende Rolle. Er war im August eingestellt worden und erwies sich als der erste Dolmetscher, der sich entschieden für die Interessen der türkischen Kollegen einsetzte und sich nicht als Handlanger der Geschäftsleitung verstand. Er war bei den Türken in kurzer Zeit sehr beliebt, nicht zuletzt, weil er sich auch um Dinge kümmerte, die mit Wohnungen, Arbeitserlaubnis etc. zu tun hatten. Allerdings war Mukadder trotz mehrfacher Warnungen der deutschen und türkischen Kollegen, mit denen er engeren Kontakt hatte, extrem unvorsichtig. Ohne sich direkt auf eine Gruppe von Kollegen, die im Betrieb organisiert arbeiten wollten, stützen zu können, lief er überall herum und verkündete selbst seinen Vorgesetzten, daß er nicht gewillt sei, die Sache der Kapitalisten zu vertreten, sondern daß er stets auf der Seite seiner Landsleute stehen würde. Festzuhalten ist jedoch, daß Mukadder ein sehr mutiger und konsequenter Interessenvertreter der türkischen Kollegen war und daß er für die zunehmend kämpferische Haltung der Kollegen eine Menge getan hat.

\section{Mukadders Entlassung und die darauf folgenden Ereignisse}

Am 15. 11. wurde dem Dolmetscher Mukadder C., aus den mit Ihnen besprochenen Gründen" (so im Kündigungsschreiben) gekündigt. „Dieser Rausschmiß richtet sich nicht nur gegen die Türken, er richtet sich überhaupt gegen die Aktivitäten von deutschen und ausländischen Kollegen in den letzten Wochen, er soll uns einschüch- 
tern" (Klassenkampf Osram vom 20. 11.).

Mukadder war vorher mehrfach von der Betriebsleitung und bezeichnenderweise auch vom Betriebsrat wegen seiner Aktivitäten für die Kollegen angegriffen und vermahnt worden. Er machte aber den Fehler, sich nicht auf die Situation bei Osram einzustellen und gerade auch im Interesse der türkischen Kollegen und im Interesse einer möglichen kontinuierlichen Arbeit im Betrieb weniger demonstrativ aufzutreten. Er schätzte seine Lage offensichtlich falsch ein und legte es auf einen Konflikt mit der Geschäftsleitung an, in der festen Überzeugung, die Solidarität der türkischen Kollegen würde eine Kündigung verhindern. Doch seine Beliebtheit bei den Kollegen machte ihn für die Betriebsleitung immer mehr zu einem gefährlichen Mann. Gleichzeitig mit seiner Kündigung erhielt er Hausverbot. Er verabschiedete sich bei den ihn aus dem Werk führenden Personalchefs mit den Worten:,Die Antwort auf meine Kündigung erhalten Sie von den Arbeitern. ${ }^{66}$ Der Betriebsrat hatte die Kündigung sofort unterschrieben, ohne vorher mit Mukadder zu sprechen.

Nach seiner Kündigung machte Mukadder seinen zweiten Fehler. Er hieit sich nicht an die deutschen und türkischen Kollegen, mit denen er im Betrieb zusammengearbeitet hatte und für die er sich auch eingesetzt hatte, sondern, "übergab den Fall dem AO-Ableger Ro, Rote Hilfe e. V.“, mit der er über das FORD-Solidaritätskomitee schon vorher Kontakt hatte. Diese hatte von den Bedingungen bei Osram keinen blassen Schimmer und wollte Mukadder als „Berliner Baha Targyn ${ }^{\text {“6 }}$ herausbringen. Als Mukadder auch zu einigen Osram-Kollegen Kontakt aufnahm, waren gleichzeitig mehrere ,Rote Hilfe e. V."Funktionäre da, die den Kollegen im Betrieb vorschreiben wollten, was sie zu tun hätten.

Wir wollen hier klarstellen, daß wir nicht glauben, man dürfe einzig und allein auf die Aktivitäten bauen, die unmittelbar aus dem Betrieb heraus möglich sind. Klar, daß wir in der gegenwärtigen Phase der Entwicklung der Klassenkämpfe auf Unterstützung ,von außen" angewiesen sind. Wir halten es nicht grundsätzlich für falsch, wenn etwa Studenten vor Betrieben agitieren. Auch die Verteiler des, „Klassenkampf Osram" sind zum Teil Studenten. Nur muß man sich ganz klar sein über die Voraussetzungen und Bedingungen, unter denen von außen eingegriffen werden kann. Was von außen kommt, muß eine ganz konkrete Unterstützung dessen sein, was in der Fabrik an realer Bewegung da ist, und was auch in der Fabrik von den Kollegen weitergetrieben werden kann. Forderungen, die von außen hineingetragen werden, oder Vorgehensweisen - „Anleitungen “ -, die draußen konzipiert sind, blockieren oder zerschlagen den ganzen Kampf, den die Kollegen im Werk führen, auf jeden Fall, wenn sie ohne exakte Einbeziehung der Situation im Werk, d. h. ohne Mitarbeit der aktiven Kollegen entwickelt werden.

Noch am Tage seiner Kündigung fing Mukadder an, die Kollegen, die zur Arbeit kamen oder nach Hause gingen, vor dem Tor über seine Kündigung und über die Hintergründe zu informieren. Das war eine richtige Aktion, denn für die Kollegen war er ja noch, ,drin" - Kündigung und Hausverbot des beliebten Dolmetschers kam für sie wie der Blitz aus heiterem Himmel. Besonders die türkischen Kollegen im Werk waren über die Kündigung empört, diskutierten über diese Sauerei und fingen an, für die Wiedereinstellung von Mukadder Unterschriften zu sammeln. D as Ziel der Kollegen, auch der aktiveren deutschen Kollegen, war es, mit dieser Unter- 
schriftensammlung der Geschäftsleitung ein Ultimatum zu stellen. Auf einer Versammlung, die zum Zeitpunkt des Ultimatums stattfinden sollte, sollte die Geschäftsleitung Farbe bekennen. Somit wäre nach Meinung der Kollegen eine Basis vorhanden gewesen, gegen die Kündigung gegebenenfalls auch mit einer Arbeitsniederlegung zu protestieren. Vor einer offiziellen Stellungnah̆me wollten die türkischen Kollegen keine Aktion starten. Ein türkischer Kollege hat das treffend so formuliert: „Wenn Firma Nein und Betriebsrat Nein, dann sagen die Arbeiter auch Nein!"

Eine solche Versammlung wäre der richtige Zeitpunkt für Mukadder gewesen, sich über das Hausverbot hinwegzusetzen, ins Werk zu kommen - was jederzeit unbemerkt möglich ist - und zu den versammmelten Kollegen zu sprechen. Auf diesen Plan gingen weder Mukadder noch die , Rote Hilfe e. V." ein. Sie hatten ihr eigenes Konzept fernab von der realen Situation bei Osram entwickelt. Dieses Konzept lief darauf hinaus, daß Mukadder an einem der folgenden Tage zusammen mit einer großen Zahl von Kollegen ins Werk gehen und dort eine Versammlung und einen Streik organisieren sollte.

Inzwischen war unter den türkischen Kollegen große Verwirrurig aufgetreten, die ihre Unterschriftensammlung erstmals zum Stocken brachte und überhaupt $\mathrm{zu}$ einer Lähmung der Aktivitäten führte. Denn Mukadder tauchte vor dem Werkstor vor jedem Schichtwechsel, bei Schichtbeginn und -schluß auf, mit einer Reihe von „,Rote Hilfe e. V.“-Funktionären, die die Kollegen permanent zu Aktionen und zum Streik aufforderten, ohne daß die Kollegen überhaupt wußten, wer diese „F reunde" Mukadders überhaupt waren. Sie waren zwar nach wie vor auf Mukadders Seite, wollten aber mit seinen „Freunden" nichts zu tun haben, weil sie nicht wußten, was die überhaupt bei Osram zu suchen hatten und was sie vorhatten. In dieser Verwirrung ging auch die Unterschriftenliste ,unter“: keiner der türkischen Kollegen, die Unterschriften gesammelt hatten, wußte mehr zu sagen, wo sie steckte. Anstatt wie geplant von einer Delegation türkischer Kollegen als Ultimatum beim Betriebsrat und bei der Geschäftsleitung abgegeben werden zu können, hatten Mukadder und die ,Rote Hilfe e. V," sie an sich gerissen und auf Flugblättern aufgefordert, weitere Unterschriften an sie zu schicken.

Die Verwirrung und Lähmung der Aktivität bei den türkischen Kollegen zeigte sich auch bei den Versammlungen, die die "Rote Hilfe e. V. ${ }^{6}$ durchfuhrte. Waren auf der ersten Versammlung noch ca. 10 Osram-Kollegen anwesend, bröckelte diese Zahl mit jeder weiteren Sitzung weiter ab. Das hielt aber die ,Rote Hilfe e. V.“ nicht $a b$, großsprecherisch ein „Osram-Solidaritätskomitee“ zu gründen, in dem nach kurzer Zeit die ,Rote Hilfe e. V."-Funktionäre unter sich waren.

In der ersten Versammlung, wo dieses Komitee auch gegründet werden sollte und wo noch einige Osram-Kollegen anwesend waren, wurde auf die Vorschläge der Kollegen überhaupt nicht eingegangen, sondern die ,Rote Hilfe e. V." setzte gegen die Bedenken der Kollegen ihre Vostellung vom Kampf bei Osram durch und machte völlig unrealistische Vorschläge, die sich vor allem am Streik bei FORD/Köln orientierten. Jeder Versuch, die Lage bei Osram realistisch einzuschätzen, wurde yon ihnen zurückgewiesen. Den Bedenken der Kollegen hielten die Funktionäre immer wieder ihre eigene Kampferfahrungen ent $t_{k}$ egen, ohne daß sie 
sagen konnten, wo sie diese Erfahrungen gesammelt hätten. Ihre Vorschläge liefen auf einen Streik hinaus, der vor dem Tor schon organisiert werden müßte, so daß daß man gar nicht erst anfängt, zu arbeiten. Dieser Vorschlag, der sich abstrakt ja großartig anhörte, bloß mit den Möglichkeiten bei Osram nichts zu tun hatte, wurde aufrechterhalten in einer Situation, wo bereits klar war, daßs in dem ,Solidaritätskomitee" keine Kollegen mitarbeiteten und im Betrieb die Stimmung schwankte zwischen Verwirrung und Passivität (bei den Türken) und offener Ablehnung gegen die Streikvorschläge, die den Kollegen pausenlos unterbreitet wurden (Ablehnung vor allem bei deutschen Kollegen). Ungeachtet dieser Lage bliesen die AO-Anhänger am Dienstag, 20.11. 1973, zum Sturm - trotz mehrfacher Warnungen von Kollegen, die die Lage bei Osram kannten.

Dieser ,Sturm ${ }^{6}$ war so geplant, daß Mukadder mit den türkischen Kollegen, die er vor dem Tor und vor Schichtbeginn morgens um sich scharen sollte, ins Werk hineingehen und dort eine Versammlung mit den Kollegen herbeiführen sollte, die entweder zu seiner Wiedereinstellung oder zum Streik führen sollte. In der Realität sah es aber am Dienstagmorgen so aus: Dem Dolmetscher gelang es nicht, mehr ais fünf Kollegen um sich zu scharen. Die anderen gingen ins Werk, ohne mit Mukadder und seiner Aktion etwas zu tun haben zu wollen. Das hielt Mukadder und seine Freunde von der $A O$ aber nicht ab, ihren einmal gefaßten Plan doch noch in die Tat umzusetzen. Mukadder ging also mit einer Handvoll von Kollegen und mit Megaphon ins Werk und da in den Pausenraum, wo vor Schichtbeginn viele türkische Kollegen ihren Kaffee oder Tee trinken. An diesem Morgen waren aber auffällig wenig Türken im Pausenraum, ca. 25. Vor diesen Kollegen stellte sich Mukadder auf den Tisch, holte das Megaphon heraus und begann auf türkisch und deutsch Reden, Parolen und Lieder anzustimmen. Er agitierte in derselben Weise, wie man es bei FORD/Köln von den dortigen Streikführern kannte, bloß mit dem Unterschied, daß die einige Tausend Kollegen vor sich hatten, während es hier nur 25 waren, von denen einige bei Arbeitsbeginn noch weggingen, also keine Anstalten machten zu streiken. Eine Ausweitung der Aktion war gar nicht möglich, weil der Pausenraum weitab von der Produktionshalle liegt, die meisten Kollegen also gar nicht mitkriegten, was sich da im Pausenraum abspielte.

Der Schichtleiter holte sofort die Polizei, Vorarbeiter, Meister und Betriebsräte notierten sich die Namen der Kollegen, die bei Mukadder im Pausenraum waren. Die Polizei kam erst zu zweit, holte dann Verstärkung, und schließlich schlugen 12 Bereitschaftspolizisten in schweren Helmen und mit Knüppeln die kleine Versammlung auseinander. Dabei gab es eine kurze Rangelei um das Megaphon und den Dolmetscher zwischen einigen Türken und den Polizisten. Vier Türken wurden zusammen mit dem Dolmetscher von der Polizei aus dem Werk geschleppt und vorläufig festgenommen. Der Mut der türkischen Kollegen, die Mukadder vor der Polizei schützen wollten, fand kein solidarisches Handeln bei den Kollegen, die das Ganze beobachtet hatten. Diese Kollegen waren auf die Aktion Mukadders überhaupt nicht vorbereitet gewesen, hielten das Ganze in dieser Form sowieso für sinnlos und sahen auch keine Möglichkeit, die Aktion auf sinnvolle Weise zu unterstützen. Das auf dem Werksgelände postierte Polizeiaufgebot aus sechs Mannschaftsbzw. Streifenwagen wurde erst abgezogen, als sich der Werksleiter persönlich verge- 
wissert hatte, daß überall im Werk gearbeitet wurde und keine offene Unruhe mehr zu erkennen war.

Das Ergebnis der Aktion waren zunächst vier festgenommene türkische Kollegen, denen sofort fristlos gekündigt wurde. Auch diese Kündigung unterschrieb der Betriebsrat sofort ohne Anhörung der Betroffenen oder ihrer Kollegen. Während des ganzen Tages, bis in die nächste Schicht hinein, gingen Polizisten in Zivil und Mitglieder der Betriebsleitung durch die Fabrik, beobachteten die Kollegen und holten noch einen Türken raus, der angeblich bei der Rangelei mit der Polizei dabeigewesen sein sollte. Auch der wurde fristlos gekündigt und aus dem Werk geführt.

Angesichts der permanenten Überwachung und des Polizeieinsatzes verbreitete sich bei den türkischen Kollegen Angst und Resignation. Bei einigen, auch bei deutschen Kollegen, herrschte zwar Empörung über den Polizeieinsatz, weil sie aber auch die Aktion des Dolmetschers für völlig idiotisch hielten, wuchs aus der Wut gegen die Polizei keine Solidarität oder ein gemeinsamer Protest gegen die Anwesenheit der Polizei. Die festgenommenen Kollegen wurden nach einigen Stunden einschließlich des Dolmetschers mit Hilfe eines Anwalts der „Roten Hilfe e. V.“ wieder freigelassen.

Mit diesem Alleingang Mukadders und der AO-Leute, die vor dem Tor dazu aufgerufen hatten, war für die Kollegen im Betrieb die letzte Chance, von innen wirkungsvoll etwas gegen die Entlassung Mukkaders zu machen, zerstört worden. Überhaupt war es in der nächsten Zeit sehr schwierig, mit mehreren Kollegen über ein einheitliches Vorgehen zu diskutieren. Pausenlos patrouillierten Abteilungsleiter und Leute von der Betriebsleitung durch die Fabrik, die Meister hielten sich ständig in der Nähe der Kollegen auf. Diese Einschüchterungsmaßnahmen hatten Erfolg. Keiner wollte in dieser Situation weitere Entlassungen riskieren, die sowieso niemandem etwas genützt hätten. Außerdem sah keiner einen Sinn in Solidaritätsbekundungen für den Dolmetscher, der sich einen Dreck um die Ratschläge der Kollegen kümmerte und nur noch auf die Funktionäre der ,Roten Hilfe e. V.“ hörte. Das war auch der eigentliche Grund, warum es nicht möglich war, vom Betrieb aus und mit Hilfe der aktiven Kollegen eine Aktion vorzubereiten, die viel mehr erreicht hätte, als das Vorgehen der ,Roten Hilfe e. V.“. Denn man hät te das auch gegen die Vorschläge des Dolmetschers machen müssen, mit dem sich die Kollegen ja gerade solidarisieren sollten. So entstand gerade für die aktiven Kollegen bei Osram eine völlig vermasselte Situation, die eigene Aktivitäten verhinderte. Nach dieser eindeutigen Niederlage für das Vorgehen der ,Roten Hilfe e. V." (die nicht darin bestand, daß Mukadder und einige Kollegen festgenommen wurden, sondern darin, daß sein Vorpreschen von den Kollegen weder verstanden noch unterstiutzt wurde), lief die Propaganda der AO erst richtig los.

An der Universität wurde zur Solidarität mit den kämpfenden Osram-Arbeitern aufgerufen. Zur Mittagsschicht versammelten sich ca. $150 \mathrm{KSV}$ Studenten vor dem Tor mit Transparenten und Mukadder an der Spitze. Sie riefen zu einer Protestdemonstration auf für Mukadder und gegen den Polizeieinsatz. An dieser Demonstration beteiligte sich kein einziger Kollege, zumal alles direkt vor dem Werkstor, d. h. vor den Augen der Geschäftsleitung stattfand. Die Taktik der AO war 
dabei, daß sie ihren Studenten vorgaukelte, đraußen vor dem Tor hätten sich andere Kollegen zu ihrer Unterstützung eingefunden. Mit den KSV Studenten ging der Demonstrationszug zu den benachbarten Siemens-Werken, wo ohne Erfolg, d. h. ohne Beteiligung von Siemens-Kollegen, versucht wurde, anhand der Osram-Vorfälle zu agitieren. In dem, Rote Hilfe e. V."-Flugblatt vom 22.11.1973 liest sich das dann so: ,Nachmittags versammeiten sich über 200 Menschen (!) mit Mukadder an der Spitze vor dem Betrieb zu einer Protestkundgebung. Anschließend bildeten sie einen Demonstrationszug bis zu den Siemens-Werken, wo sie die Siemens-Kollegen von den Vorfällen unterrichteten."

Das war aber noch nicht alles. An den folgenden Tagen wurde vor verschiedenen Werken, auch vor den drei anderen Berliner Osram-Werken, und vor den Berufsschulen ein Flugblatt verteilt, in dem es zu den Aktivitäten der Osram-Kollegen nach Mukadders Kündigung heift: ,Sie sammelten 600 Unterschriften, legten am Freitag für eine Stunde die Arbeit nieder, führten am Montag eine Pausenversammlung durch. Am Dienstagmorgen holten die Kollegen Mukadder in den Betrieb hinein und zeigten auf einer Versammlung in der Kantine ihre Entschlossenheit, für die sofortige Wiedereinstellung zu kämpfen. Daraufhin ließ die Geschäftsleitung die Kantine durch 20 Bereitschaftspolizisten stürmen. Die Polizisten griffen brutal an, schlugen mit Knüppeln auf die Arbeiter ein und verhafteten Mukadder und vier andere Kollegen...Mukadder wurde die Ausweisung angedroht, alle verhafteten Kollegen und noch 23 andere wurden fristlos entlassen." (Flugblatt vom 22.11. 1973)

Von diesen Angaben ist alles bis auf die Schilderung der Polizeiaktion gelogen. Weder wurden 600 Unterschriften gesammelt (es waren knapp über 200), weder legten die Kollegen am Freitag für eine Stunde die Arbeit nieder (in einer Abteilung wurde bei Schichtbeginn über die Kündigung Mukadders diskutiert; das dauerte - bis zum Eintreffen des Meisters - eine halbe Stunde), weder wurde eine Pausenversammlung durchgeführt (in der Pause wurde wie immer unter den Kollegen diskutiert), und was es mit der Versammlung in der Kantine auf sich hatte, haben wir schon berichtet. Diese Versammlung von ca. 25 Kollegen, die zum Teil nur zufällig da waren, weil sie Kaffee trinken wollten, wurde in der Roten Fahne sogar zu einer Betriebsversammlung hochgejubelt (Überschrift in der Roten Fahne vom 28. 11. $1973 \mathrm{zu}$ den Osram-Vorfällen: ,SPD-Polizei zerschlägt Betriebsver" sammlung!"). Auch die im Flugblatt angegeben Zahl von 23 weiteren Entlassungen war eine Falschmeldung. Außer den vier Festgenommenen und dem einen Kollegen, der später von der Polizei ,entlarvt ${ }^{t}$ wurde, wurde noch eine Kollegin am selben Tag aus anderen Gründen entlassen, die mit den Vorfällen am Dienstagmorgen im Pausenraum nichts zu tun hatten. Die AO hat te es mit ihrer revolutionäten Taktik also ,bloß ${ }^{6}$ zu 5 Entlassungen gebracht. Eine Reihe anderer türkischer Kollegen wurde mündlich verwarnt.

Bezeichnenderweise wurde dieses mit maßlosen Übertreibungen und Lügen gespickte Flugblatt nicht vor unserem Werk verteilt, wo die Kollegen ja genau wußten, was passiert war, sondern nur vor anderen Betrieben und Berufsschulen, wo die Kollegen nichts von der wirklichen Situation wußten. Das beweist, daß Lügen und Ubertreibungen nicht zufällig und aus Schlamperei, sondern mit voller Absicht verbreitet wurden, mit dem Ziel, die Aktivitäten der AO und ihrer ,Roten 
Hilfe e. $\mathrm{V} .{ }^{66}$ groß herauszustellen und den Kollegen und Studenten an der Uni vorzugaukeln, bei Osram wäre unter ihrer Führung ein großer Kampf ausgebrochen.

Trotz der niederschmetternden Erfahrungen und trotz des Unheils und der Verwirrung, die die $\mathrm{AO}$ jetzt schon unter den Osram-Kollegen und vor allem unter den Türken angerichtet hatte, und trotz der Tatsache, daß sich schon jetzt einige der am Dienstag fristlos entlassenen türkischen Kollegen von der „Roten Hilfe e. $V_{0}$ " klar distanziert hatten, erwiesen sich die ,erfahrenen Kämpfer" der $\mathrm{AO}$ als blinde Opfer ihrer eigenen Propaganda und riefen zu neuen Aktionen auf. Am Donnerstag verbreiteten sie unter Kollegen, am Freitag würde gestreikt, und sie würden vor Schichtbeginn das Werk von außen, dicht machen'. Der Betriebsrat lief den ganzen Donnerstag rum und empfahl den Kollegen, am nächsten Morgen das Werk erst zu betreten, wenn dies ohne Schwierigkeiten möglich wäre. Sie würden die Ausfallzeit voll bezahlt bekommen! Die meisten Kollegen nahmen das dankbar zur Kenntnis, da sie weder gewillt waren, zu streiken, noch ,ihr Werk freizukämpfen".

An folgenden Morgen, 23 11. 1973, verteilte die AO vor der Frühschicht ein Flugblatt, in dem es heißt: ,Arbeiter von Osram! Treten wir heute in den Streik, um endgültig unsere Forderung nach der Rücknahme der Kündigung des türkischen Dolmetschers und die sofortige Wiedereinstellung aller entlassenen Kollegen durchzusetzen. Wir versammeln uns jetzt vor dem Tor und werden demokratisch über den Streik abstimmen. - Wir werden demokratisch unsere Forderungen beschließen. Wir werden demokratisch die Kollegen wählen, deutsche, türkische, griechische und jugoslawische, die eine Streikleitung bilden, den Streik organisieren und mit der Geschäftsleitung verhandeln.“

Angesichts der wirklichen Situation im Betrieb war dieser Aufruf lächerlich, er wurde auch von keinem Kollegen ernstgenommen. Keiner machte Anstalten, für irgende twas abzustimmen, zu streiken und vor allem eine Streikleitung zu wählen, was für die $\mathrm{AO}$ ja immer das wichtigste ist. Da nützten auch die vielleicht hundert ,Menschen ${ }^{6}$ nichts, die in Autos vor dem Werk auf eine Aktion der Kollegen warteten, um sie dann mit der Kampferfahrung und der Kraft des KSV zu stärken. Zu diesem Zeitpunkt war die Reaktion der Kollegen nicht mehr nur Verwirrung und Resignation, sondern schon völlige Interesselosigkeit und offene Ablehnung gegen diese Leute ,vor dem Tor ${ }^{6}$. Die "Rote Hilfe e. V." und ihre verbündeten Studenten zogen denn auch schon, bevor die letzten Kollegen zur Arbeit kamen, wieder ab. Betriebsrat und Geschäftsleitung nutzten die Situation zu maßloser Hetze gegen die „Kommunisten" und verbreiteten wilde Gerüchte (,die wollen das Werk stürmen und eure Autos anzünden').

In einem weiteren Flugblatt wurde mittags bei Schichtwechsel das Fehlschlagen des Streiks allein den Einschüchterungen der Meister und Geschäftsleitung in die Schuhe geschoben.Kein Wort von Selbstkritik und natürlich auch kein Wort davon, daß es ihr eigenes Vorgehen war, das die resignative und passive Haltung der Kollegen schließlich sogar gegenüber dem vorher so beliebten Dolmetscher zur Folge hatte.

Vier Tage später wurde dann auch noch ein Flugblatt verteilt, in dem die ${ }_{2}$ Rote Hilfe e. $V_{.}^{66}$ dazu aufforderte, die Kollegen sollten eine außerordentliche 
Betriebsversammlung fordern. Auch dieses Flugblatt fand überhaupt keine Resonanz mehr. Die Strategen von der AO kennen natürich ihr Betriebsverfassungsgesetz. Dort heißt es in $\S 43$, Absatz 3: „Der Betriebsrat ist berechtigt, und auf Wunsch des Arbeitgebers oder mindestens einem Viertel der Arbeitnehmer verpflichtet, eine Betriebsversammlung einzuberufen und den beantragten Beratungsgegenstand auf die Tagesordnung zu setzen". Nur wissen sie nicht, daß die zu einer außerordentlichen Betriebsversammlung benötigten Unterschriften nur im Werk zusammengebracht werden können von Kollegen, die das Vertrauen großer Teile der Belegschaft haben. Und sie hatten keinen Schimmer davon, daß eine Betriebsversammlung zu diesem Zeitpunkt politisch fatal gewesen wäre: Die Belegschaft war so gespalten, verwirrt, eingeschüchtert, mißtrauisch oder gar offen feindlich gegenüber den gekündigten Kollegen, die jetzt mit den „Menschen " vor dem Tor in einen Topf geworfen wurden, daß Geschäftsleitung und Betriebsrat hätten offen und ungestraft auftrumpfen können.

\section{Nun zu den Entlassenen}

Von den fünf entlassenen türkischen Kollegen waren mindestens zwei dabei, die mit der Aktion am Dienstagmorgen überhaupt nichts zu tun hatten. Sie waren zufällig im Pausenraum gewesen und wurden von der Polizei rausgeprügelt, ohne daß sie selber aktiv geworden wären. Ein weiterer hat sogar seine Kollegen ausdrücklich davor gewarnt, sich in die Auseinandersetzung mit der Polizei einzulassen. Er wurde dann später von der Polizei als ein ,Sprecher ${ }^{6}$ der Türken identifiziert und entlassen. Die anderen Kollegen waren zwar mit Mukadder näher bekannt, hatten aber keine Vorstellung, was eigentlich Mukadders Freunde, die AO und die „Rote Hilfe e. V.“ politisch wollten.

Das führte dann auch dazu, daß in dem besagten ,Osram-Solidaritätskomitee kein einziger der Entlassenen mitarbeitete. Gebunden waren einige von ihnen an die „Rote Hilfe e. V.“ nur noch über deren Rechtsanwalt, der vor dem Arbeitsgericht gegen ihre Kündigung Klage erhoben hatte. In Diskussionen mit Kollegen im Betrieb, türkischen und deutschen, die den Entlassenen helfen wollten, kam heraus, daß ihnen allen die politische Linie der ,Roten Hilfe e. V. "überhaupt nicht bekannt war bzw. verdächtig oder falsch vorkam.

Da die Entlassenen von dem Geld, das die ,Rote Hilfe e. V. ${ }^{66}$ mit großem Tamtam in ganz Westberlin für sie gesammelt hatte, entweder nicht wirksam unterstützt wurden, oder es überhaupt abgelehnt hatten, von der ${ }_{2}$ Roten Hilfe e. V. ${ }^{6}$ Geld anzunehmen, kamen sie mit ihren bis zu sieben Köpfen zählenden Familien in arge Bedrängnis. Das konnte nur notdürftig ausgeglichen werden von dem Geld, das Osram-Kollegen unter schwierigen Bedingungen im Betrieb sammelten. Die zu dieser Sammlung nötige heimliche Agitation hat den ersten Ansatz dazu geschaffen, die von der ,Roten Hilfe e. V. “ zerstörte Solidarität unter den Kollegen wiederherzustellen.

Den Entlassenen war ebenso unklar, wie sie gegen die Kündigung wirklich vorgehen sollten. Der ,Rote Hilfe e. $V$, "-Anwalt ließ sich zwar von dreien von ihnen 
eine Vollmacht ausstellen, redete aber nicht mit ihnen über den zu erwartenden Prozeß und uber ihre Chancen, den Prozeß zu gewinnen. Diese Unsicherheit bei den Entlassenen führte dazu, daß zwei von den Entlassenen mit ihren Vertrauensleuten, die mir der Haltung des Betriebsrats nicht einverstanden waren, zur Gewerkschaft gingen und dort Rechtsbeistand erhielten; ein anderer, der erst vor einem Monat in die Gewerkschaft eingetreten war und keinen Anspruch auf Rechtsbeistand hat te, ging zu einem anderen Rechtsanwalt. Schließlich zogen auch die zwei letzten Enlassenen ihre Prozeßvollmacht für den ",Rote Hilfe e. V. ${ }^{6}{ }^{6}$ Anwalt zurück. Somit war für sie auch die letzte Verbindung zur "Roten Hilfe e. V." gekappt.

Das alles aber hinderte diese "Rote Hilfe e. V.", die AO und das, Osram-Solidaritätskomitee "nicht, đie Entlassenen als bewuß te "Kämpfer" herauszustellen. Das geschah auf einer groß angekündigten Veranstaltung am 2.12.1973, auf der Baha Targyn, Mukadder und Funktionäre der ,Roten Hilfe e. V." großsprecherische Reden hielten, in denen abermals die Lage bei Osram mit der bei FORD/Köln gleichgestellt und Mukadder so zu einem zweiten Baha Targyn wurde. Bezeichnend für diese Großsprecherei ist, daß auf dieser Veranstaltung kein einziger Osram-Kollege da war, und auch keiner der Entlassenen. Das heiß t, jetzt mußte eigentlich auch dem letzten klar sein, daß das ,Osram-Solidaritätskomitee" ein von den Osram-Kollegen völlig abgehobenes Phantasie- und Propagandagebilde der KPD war. Den anwesenden KSV-Siudenten wurde aber dennoch vorgekaukelt, welch großartigent Kampf die KPD bei Osram fïhrt (siehe auch Rote Fahne vom 5. 12. 1973).

\section{Was ist das Ergebnis dieser $\mathrm{AO}$-Kampagne?}

Was vorher unter oft langwieriger Kleinarbeit bei Osram erreicht werden konnte, nämlich eine solidarische Bewegung unter den Kollegen, wurde zunächst kaputtgemacht. Vorherrschend waren Verwirrung über das Vorgehen Mukadders und die Ziele und Methoden seiner Freunde, und Resignation, weil der gemeinsame Widerstand, der am Anfang durchaus da war, gegen die Kündigung Mukadders durch das Vorgehen der AO zunichtegemacht wurde. Zudem hatte es die Geschäftsleitung angesichts der geradezu selbstmörderischen Taktik Mukadders und dem ebenso ahnungslosen wie verantwortungslosen Vorgehen der $\mathrm{AO}$ leicht, die Kollegen einzuschüchtern und dazu antikommunistische Vorurteile aufzubauen oder zu verstärken.

Angesichts dieser vermasselten Situation war es sehr schwer, eine von den Kollegen ausgehende Solidarität mit den entlassenen Kollegen zu organisieren. Das war gerade bei den Türken schwer, weil die jetzt am meisten verwirrt und ängstlich waren. Es blieb bei einer vorsichtigen Geldsammlung und bei bescheidener Hilfe für die Entlassenen bei der Arbeitssuche und der Beschaffung von Kleidern für deren Kinder. Die entlassenen Kollegen trifft es jetzt besonders hart, weil sie kaum Arbeit finden und Angst haben müssen, daß ihre Aufenthaltsgenehmig̊ung nicht verlängert wird. Sie müssen befürchten (wenn sie keine Arbeit finden), daß sie angesichts des jetzt einsetzenden Gastarbeiterstops in die Türkei zurück müssen. Allerdings haben sie nach Auskunft der Rechtsanwälte durchaus Chancen, ihren Arbeitsgerichtspro- 
zeß zu gewinnen. Aber bei Osram werden sie wohl kaum wieder eingestellt.

Zur AO ist festzuhalten, daß sie es mit ihrer völlig von den realen Bedingungen abgelösten Taktik innerhalb kurzer Zeit erreicht hat, eine Bewegung unter den Osram-Kollegen derart zu bremsen, daß es vermu tlich eine längere Zeit dauern wird, bis die Solidarität und die Kampfbereitschaft unter den Kollegen, besonders unter den türkischen, wieder so weit ist wie vorher. Das ist eine klare Schwäche auch für die kommende Tarifauseinandersetzung.

Aus den Aktivitäten der AO kann man ablesen, daß es ihr überhaupt nicht darauf ankommt, unter den Kollegen eine kämpferische Bewegung zu unterstitzen und die Kollegen dahin zu bringen, sich im Betrieb gegen die Angriffe der Kapitalisten zu wehren. Außerdem zeigte sich, daß die Genossen der AO uberhaupt nicht in der Lage sind, die Situation in einem Betrieb einigermaßen realistisch sinzuschätzen und entsprechend zu handeln. Nur so ist es zu erklären, daß sie die Situation bei Osram mit der bei FORD-Köln einfach gleichsetzten.

Eine weitere Fehleinschätzung, die sich niemand leisten würde, der auch nur die geringste Ahnung über die Kampfmöglichkeiten im Betrieb hat, bestand darin, daß die AO-Funktionäre meinten, allein mit einer permanenten Agitation vor dem Tor würde sich im Betrieb etwas tun.

Ihre Taktik lief daraus hinaus, durch aufgesetzte und von den Kollegen überhaupt nicht getragene Aktivitäten einige Kollegen zu exponieren, sie zu Opfern des Kapitals zu machen und dann mit diesen Opfern propagandistisch zu arbeiten. Dabei ist es ihnen völlig gleichgültig, ob diese Kollegen - wie bei Osram - über. haupt ihre eigene Lage und die Politik der AO richtig einschätzen können oder nicht. Die KPD.AO hat in jedem Fall ein Solidaritätskomitee mehr, mit dem sie sich schmücken und mit dem sie sich in der Propaganda als vorantreibende Kraft im Klassenkampf aufplustern kann. Ob dabei der reale Kampf in den Betrieben vorankommt oder kaputtgeht, ist ihr völlig gleich. Diese Politik trägt objektiv dazu bei, die Kampfkraft der Kollegen zu schwächen, organisierte Ansätze der Kollegen in den Betrieben kaputtzumachen und die antikommunistische Haltung vieler Kollegen zu stärken. 